\title{
Gestational methyl donor deficiency alters key proteins involved in neurosteroidogenesis in the olfactory bulbs of newborn female rats and is associated with impaired olfactory performance
}

\author{
Sarah El Hajj Chehadeh, Grégory Pourié, Nicolas Martin, Jean-Marc Alberto, Jean-Luc Daval†, \\ Jean-Louis Guéant*† and Brigitte Leininger-Muller* \\ Inserm U954, Faculté de Médecine de Nancy, Université de Lorraine, Vandoeuvre-lès-Nancy, F-54500, France
}

(Submitted 13 May 2013 - Final revision received 25 September 2013 - Accepted 25 September 2013 - First published online 12 November 2013 )

\section{Abstract}

Gestational methyl donor deficiency (MDD) leads to growth retardation as well as to cognitive and motor disorders in 21-d-old rat pups. These disorders are related to impaired neurogenesis in the cerebral neurogenic areas. Olfactory bulbs (OB), the main target of neuronal progenitors originating from the subventricular zone, play a critical role during the postnatal period by allowing the pups to identify maternal odour. We hypothesised that growth retardation could result from impaired suckling due to impaired olfactory discrimination through imbalanced apoptosis/neurogenesis in the OB. Since neurosteroidogenesis modulates neurogenesis in OB, in the present study, we investigated whether altered neurosteroidogenesis could explain some these effects. Pups born to dams fed a normal diet ( $n$ 24) and a MDD diet ( $n$ 27) were subjected to olfactory tests during the lactation and weaning periods ( $n 24$ and 20 , respectively). We studied the markers of apoptosis/neurogenesis and the expression levels of the key neurosteroidogenic enzyme aromatase, the cholesterol-transfer protein StAR (steroidogenic acute regulatory protein) and the ER $\alpha$ oestrogen receptor and the content of oestradiol in OB. The 21-d-old MDD female pups displayed lower body weight and impaired olfactory discrimination when compared with the control pups. MDD led to greater homocysteine accumulation and more pronounced apoptosis, along with impaired cell proliferation in the OB of female pups. The expression levels of aromatase, StAR and ER $\alpha$ as well as the content of oestradiol were lower in the OB of the MDD female pups than in those of the control female pups. In conclusion, gestational MDD may alter olfactory discrimination performances by affecting neurogenesis, apoptosis and neurosteroidogenesis in OB in a sex-dependent manner. It may be involved in growth retardation through impaired suckling.

Key words: Methyl donor deficiency: Neurosteroids: Olfactory bulbs: Olfactory discrimination

Methyl donor deficiency (MDD) leads to the accumulation of the toxic amino acid homocysteine (Hcy), which is associated with neural tube defects, intra-uterine growth retardation in the progeny ${ }^{(1)}$ and neurodegenerative diseases in adulthood ${ }^{(2)}$. The accumulation of Hcy can affect neurogenesis and synaptic plasticity during brain development ${ }^{(3)}$. Reduced neuroprogenitor proliferation and increased apoptosis occur in the fetal brain of folate-deficient rodents during late gestation ${ }^{(4)}$. Indeed, Hcy is a metabolite of the essential amino acid methionine and can either be remethylated to methionine by enzymes requiring folate or vitamin $\mathrm{B}_{12}$ or be catabolised by cystathionine $\beta$-synthase to generate cysteine. In an animal model based on rats born to dams fed a MDD diet during gestation and lactation $^{(5-8)}$, hallmarks of increased apoptosis were observed in selective brain structures of the 21-d-old progeny including the cerebellum and the pyramidal neurons of Cornu Ammonis (CA1) region as well as the main areas of persisting brain neurogenesis, i.e. the dentate gyrus in the hippocampus and the subventricular zone. The 21-d-old animals exhibited locomotor coordination impairment and cognitive defects, which mostly persisted in adulthood despite the restoration of normal diet feeding after weaning. Deficient pups also displayed growth retardation with dramatically reduced body weight ${ }^{(5,6)}$ and visceral manifestations of fetal programming ${ }^{(9,10)}$. We had also reported that some of these deleterious effects on growth could be linked to the MDD

Abbreviations: ER, oestrogen receptor; Hcy, homocysteine; MDD, methyl donor deficiency; OB, olfactory bulbs; SAH, S-adenosylhomocysteine; SAM, S-adenosylmethionine; StAR, steroidogenic acute regulatory protein.

*Corresponding authors: J.-L. Guéant, fax +33 3836832 79, email jean-louis.gueant@univ-lorraine.fr; B. Leininger-Muller,

email brigitte.leininger@univ-lorraine.fr

† These authors contributed equally. 
consecutive remodelling of gastric cellular organisation leading to ghrelin dysfunction ${ }^{(11)}$. This hormone plays a significant role in growth through its dual role as a growth hormonereleasing factor and as an orexigenic peptide ${ }^{(12)}$

Apoptosis in selective brain structures could also be involved in abnormal feeding behaviour and explain the dramatic growth retardation of MDD pups during the suckling period. Indeed, feeding disorders could be related to impaired olfactory discrimination, which plays a central role during the breastfeeding period. Early olfactory experience is critical for survival, and olfactory bulbs (OB) play a critical role during the postnatal period by allowing the pups to identify maternal odour ${ }^{(13,14)}$. Newborn rats rapidly learn to identify maternal odours following the binding of noradrenaline to $\beta$-adrenoreceptors in the OB resulting from tactile stimulation ${ }^{(15)}$. OB are defined as postnatal neurogenesis areas because subventricular zone neuronal progenitors migrate to these areas via the rostral migratory stream where they differentiate into bulbar interneurons. In rodents, the increased neurogenesis occurring in $\mathrm{OB}$ during pregnancy and the lactation period is essential for maternal care and offspring recognition ${ }^{(16)}$

Neurogenesis is closely related to neurosteroidogenesis. Besides their classical association with the neuroendocrine regulation of reproduction and sexual behaviour, most of the so-called neurosteroids originating from local brain synthesis are involved in developmental and adult cerebral plasticity $^{(17)}$. The synthesis of neurosteroids involves the recruitment of the rate-limiting step StAR protein (steroidogenic acute regulatory protein), which transfers cholesterol into the mitochondrial inner membrane to reach the cholesterol side-chain cleavage enzyme (CYP11A1) to transform it into pregnenolone ${ }^{(18,19)}$. StAR is widely distributed in the adult brain, especially in the neurogenic areas including $\mathrm{OB}^{(20,21)}$. The androgen-to-oestrogen-converting enzyme cytochrome P450 aromatase (CYP19) represents the key enzyme of neurosteroidogenesis ${ }^{(22)}$. The resulting oestradiol can modulate neurogenesis in the subventricular zone ${ }^{(23)}$ by allowing the recruitment of newborn cells in OB. This bears out the influence of oestradiol in olfactory discrimination.

In this context, in the present study, we investigated whether gestational MDD could affect olfactory performance through increased apoptosis and lower rates of neurogenesis in OB. For this purpose, 21-d-old rats born to dams fed a MDD diet and those born to dams fed a normal diet during the gestation and lactation periods were subjected to behavioural tests evaluating olfaction. Later, for the first time, in association with the classical determinants of Hcy metabolism, the expression levels of aromatase and the cholesterol-transfer protein StAR as well as oestrogen receptor (ER) $\alpha$ and the consecutive oestradiol content were monitored in the $\mathrm{OB}$ of the MDD pups and compared with those of the control pups.

\section{Materials and methods}

\section{Animals}

Experiments were carried out in Wistar rats (Janvier) in accordance with the National Institutes of Health Guide for the Care and Use of Laboratory Animals. Adult female rats were maintained under standard laboratory conditions, under a $12 \mathrm{~h}$ light $-12 \mathrm{~h}$ dark cycle, with access to food and water ad libitum. The rats were fed either a standard diet (Maintenance diet M20; Scientific Animal Food and Engineering) or a folate and choline-deficient diet 1 month before pregnancy, as described previously ${ }^{(6,7)}$ (Special Diet Service). The assigned diets were fed until weaning of the offspring at postnatal day 21 .

\section{Behavioural evaluation of olfaction}

To monitor the acquisition of olfactory recognition in pups of dams fed the MDD diet ( $n$ 27) or the normal diet ( $n$ 24), their ability to return to their home cage successfully by means of environmentally sensitive information was recorded in a T-maze between postnatal days 5 and $19^{(24)}$. The animal model of gestational MDD is standardised and is widely used in different research projects. The differences between the number of weighed animals and that finally included in the behavioural tests are explained by the fact that a few of the animals were used for other protocols in the laboratory.

At each time point of postnatal development (see the Results section), each pup was subjected to a one-trial test consisting in behaving freely in a T-maze area (two arms of $30 \mathrm{~cm}$ length, walls of $10 \mathrm{~cm}$ height and a corridor of $5 \mathrm{~cm}$ width). The home cage (without the mother rat) of each pup was positioned at the end of one arm and a clean cage of the same size was positioned at the end of the other arm. These two cages were randomly placed between the right and left sides of the maze, and the corridors were carefully washed after each experiment. The test was considered successful when a pup returned directly to its home cage without visiting the arm containing the clean cage or without returning to the starting arm. The maximum time allowed for a pup to return to its home cage was $2 \mathrm{~min}$. The time taken by the pups to reach the home cage was recorded. If a pup did not find its home cage within the allocated time, the maximum time (2 min) was affected.

Discrimination abilities at the time of weaning were tested through a place-preference test carried out in 21-d-old MDD ( $n$ 20) and control ( $n$ 24) pups. In accordance with previous studies $^{(25,26)}$, avoidance response to the irritant odour of toluene was evaluated in a corridor maze $(80 \mathrm{~cm}$ in length, $8 \mathrm{~cm}$ in breadth and $15 \mathrm{~cm}$ in height). Both ends of the corridor contained a watch glass with a filter paper soaked in $15 \mu \mathrm{l}$ of either pure toluene or distilled water (used as control). Toluene odour and water were randomly distributed in the right and left sides of the maze during each test. The odorant compartments were closed with a grid to avoid contact and covered to avoid the dispersion of odour. Each pup was placed in the middle of the corridor, facing a wall, and then allowed to move freely for $5 \mathrm{~min}$. The movements of the pups were video-recorded and analysed with a video tracking system for automation of the behavioural experiments (View Point). Data on the total duration spent by each pup in each part of the corridor divided into three equal zones, defined as the 
repulsive toluene zone, the neutral central zone and the control water zone, were collected.

\section{Tissue collection}

Rat pups were weighed and killed at postnatal day 21 with an overdose of isoflurane. Whole brains were rapidly harvested. For immunohistochemical analyses, brains were immediately frozen in methylbutane previously chilled to $-30^{\circ} \mathrm{C}$ and stored at $-80^{\circ} \mathrm{C}$. For biochemical analysis, brains were rapidly collected. The $\mathrm{OB}$ were dissected before freezing in liquid $\mathrm{N}_{2}$ and stored at $-80^{\circ} \mathrm{C}$.

\section{Isolation of mitochondrial and microsomal fractions}

The mitochondrial and microsomal fractions of $O B$ were isolated according to the following protocol. The samples were homogenised ( $1 \mathrm{~g}$ of tissue $/ 3 \mathrm{ml}$ buffer) in the isolation medium (0.32 $\mathrm{m}$-sucrose, $10 \mathrm{~mm}$-Tris- $\mathrm{HCl}$ and $1 \mathrm{~mm}$-EDTA, $\mathrm{pH} 7 \cdot 4)$. The homogenates were centrifuged at $2000 \mathrm{~g}$ for 3 min at $4^{\circ} \mathrm{C}$ (TLX ultracentrifuge OptimaTM, Beckman), and the pellet containing nuclei and cell debris was discarded. Centrifugation of the supernatant at $12500 \mathrm{~g}$ for $10 \mathrm{~min}$ at $4^{\circ} \mathrm{C}$ resulted in a new supernatant, which was retained for the isolation of microsomes. The pellet was resuspended in the isolation medium and centrifuged at $11000 \mathrm{~g}$ at $4^{\circ} \mathrm{C}$ for $10 \mathrm{~min}$. The mitochondrial fraction was pelleted by additional centrifugation at $10000 \mathrm{~g}$ for $10 \mathrm{~min}$ and suspended in radioimmunoprecipitation assay (RIPA) buffer $(136 \mathrm{~mm}-\mathrm{NaCl}$, $1.7 \mathrm{~mm}-\mathrm{KH}_{2} \mathrm{PO}_{4}, \quad 10 \mathrm{~mm}-\mathrm{Na}_{2} \mathrm{HPO}_{4}, \quad 1 \%$ Tergitol (v/v), $0.5 \%$ deoxycholate (w/v), $0 \cdot 1 \%$ SDS (w/v), $100 \mathrm{~mm}-\mathrm{Na}_{3} \mathrm{VO}_{4}$, $57 \mathrm{~mm}$-phenylmethylsulfonyl fluoride and $0 \cdot 14 \%$ protease inhibitor) such that the mitochondrial fraction extracted from $0.1 \mathrm{~g}$ of brain tissue was suspended in $0.5 \mathrm{ml}$ of RIPA buffer.

For the isolation of microsomal fractions, the supernatant collected previously was centrifuged at $15000 \boldsymbol{g}$ for $20 \mathrm{~min}$ at $4^{\circ} \mathrm{C}$; the pellet was discarded and the supernatant was centrifuged again at $105000 \mathrm{~g}$ for $90 \mathrm{~min}$ at $4^{\circ} \mathrm{C}$. The microsomal pellet was resuspended in the storage buffer $(80 \mathrm{~mm}-$ $\mathrm{K}_{2} \mathrm{HPO}_{4}, \quad 19 \mathrm{~mm}_{-} \mathrm{KH}_{2} \mathrm{PO}_{4}, \quad 0 \cdot 2 \mathrm{~mm}-\mathrm{EDTA}$ and $20 \%$ glycerol $(\mathrm{v} / \mathrm{v}), \mathrm{pH} 7 \cdot 4)$ such that $1 \mathrm{ml}$ of suspension contained a microsomal fraction extracted from $3 \mathrm{~g}$ of tissue. The protein concentration of the subcellular fractions was assessed using a bicinchoninic acid protein determination kit (Interchim) with bovine serum albumin as a standard.

Measurement of vitamin $B_{12}$, folate,

$\mathrm{S}$-adenosy/methionine and S-adenosy/homocysteine concentrations

The concentrations of vitamin $\mathrm{B}_{12}$ and folate were measured using a radioisotope dilution assay (simulTRAC-SNB; ICN Pharmaceuticals) as described previously ${ }^{(27)}$. The concentrations of the classical determinants of Hcy metabolism in tissue homogenates were measured using HPLC adapted from Luippold et $a l^{(28)}$. S-Adenosylmethionine (SAM) is demethylated to form $S$-adenosylhomocysteine (SAH) and $\mathrm{Hcy}$ is generated from the hydrolysis of SAH. Proteins were precipitated with $0 \cdot 2 \mathrm{M}-\mathrm{HClO}_{4}$ before injection into the column (Lichrospher, $100 \mathrm{RP}-\mathrm{C} 18,5 \mu \mathrm{m}, 250 \times 4 \mathrm{~mm}$ inner diameter; Interchim). The mobile phase, consisting of $50 \mathrm{~mm}$-sodium phosphate ( $\mathrm{pH} \mathrm{3} 2$ ), $10 \mathrm{~mm}$-heptane sulfonate and acetonitrile (10-20\% from 0 to $20 \mathrm{~min}$ ), was applied at a flow rate of $0.75 \mathrm{ml} / \mathrm{min}$. The concentrations of SAM and SAH were quantified using a UV detector set at $254 \mathrm{~nm}$.

\section{DNA methylation}

Global DNA methylation was quantified in the tissular extracts of OB obtained from male ( $n$ 5) and female ( $n$ 5) pups of the MDD diet-fed dams and pups of the normal diet-fed dams ( $n$ 5, respectively) using the Methylamp ${ }^{\text {TM }}$ Global DNA Methylation Quantification Kit (Epigentek, Euromedex), as described previously ${ }^{(29)}$. Briefly, the methylated fraction of DNA is recognised by a 5-methylcytosine antibody and quantified through an ELISA-like reaction. The amount of methylated DNA is proportional to the intensity of absorbance.

\section{Immunohistochemical analyses}

Immunohistochemical analyses were carried out on cryostatgenerated $20 \mu \mathrm{m}$ sagittal brain sections mounted onto glass slides. For immunostaining, tissue sections previously fixed in $4 \%$ paraformaldehyde (Sigma) were kept for $15 \mathrm{~min}$ at room temperature and were incubated for $10 \mathrm{~min}$ in PBS solution containing $0 \cdot 1 \%$ of Triton X-100. After washing the tissue sections three times with PBS for $5 \mathrm{~min}$, non-specific binding was blocked with bovine serum albumin $(10 \%, \mathrm{w} / \mathrm{v})$ dissolved in PBS and incubation was carried out for $1 \mathrm{~h}$. The tissue sections were incubated for $72 \mathrm{~h}$ at $4^{\circ} \mathrm{C}$ with polyclonal rabbit antibodies against Hcy (1:200 dilution; Millipore), StAR (1:200 dilution; kindly provided by Professor D. Stocco, Texas Tech University), aromatase (1:200 dilution; BioVision Research Products) and oestradiol (1:200 dilution; US Biological) or a polyclonal mouse antibody against ER $\alpha$ (1:200 dilution; Santa Cruz Biotechnology). We used the monoclonal rabbit cleaved anti-caspase 3 antibody (1:200 dilution; Cell Signaling Technology) for the specific detection of apoptosis. The expression of the Ki-67 protein specifically associated with cell proliferation was analysed in $\mathrm{OB}$ sections using a polyclonal rabbit antibody against Ki67 (1:200 dilution; Abcam).

The tissue sections were then incubated for $1 \mathrm{~h}$ at room temperature in the presence of a secondary antibody (donkey anti-rabbit or donkey anti-mouse $\operatorname{IgG}$ conjugated to Alexa Fluor, 1:2000; Molecular Probes).

Cell nuclei were stained with the fluorescent dye 4,6-diamidino-2-phenylindole $(5 \mu \mathrm{g} / \mathrm{ml}$ in distilled water). The tissue sections were then washed three times with PBS, coverslipped using Fluoromount mounting medium (Sigma) and kept in the dark until fluorescence analysis. The tissue sections were observed with a fluorescence microscope (Olympus) at $\times 20$ magnification. The pictures were digitised with a monochromatic camera and analysed using the cellF software (Olympus). 
Table 1. Body weight $(\mathrm{g})$ at weaning of 21-d-old rat pups born to dams fed a methyl donor-deficient (MDD) or a normal diet (Mean values and standard deviations, $n 20$ pups per group)

\begin{tabular}{llllll}
\hline & \multicolumn{2}{c}{ Females } & & \multicolumn{2}{c}{ Males } \\
\cline { 2 - 3 } \cline { 6 - 6 } & Mean & SD & & Mean & SD \\
\hline Control & 39.72 & 3.08 & & 39.25 & 4.10 \\
MDD & $17.32^{\text {*** }}$ & 4.39 & & $21.86^{* * *}$ & 4.85
\end{tabular}

***Mean values were significantly different from the control rat pups for each sex $(P<0.0001$, ANOVA $)$

\section{Western blot analyses}

The dissected cerebral regions were solubilised in RIPA lysis buffer containing $140 \mathrm{~mm}-\mathrm{NaCl}, 0.5 \%$ (w/v) sodium deoxycholate, $1 \%(\mathrm{v} / \mathrm{v})$ Nonidet P-40, $0 \cdot 1 \%(\mathrm{w} / \mathrm{v})$ SDS and protease inhibitors (Roche Applied Science), lysed by three cycles of freezing/thawing and finally centrifuged at $4{ }^{\circ} \mathrm{C}$ for $30 \mathrm{~min}$ at $15000 \mathrm{~g}$. The concentrations of proteins in the supernatant were determined using the BCA Protein Assay Kit (Interchim). Samples were mixed with an equal volume of $2 \times$ Laemmli buffer and denatured by heating for $5 \mathrm{~min}$ at $100^{\circ} \mathrm{C}$.

Mitochondrial fractions $(30 \mu \mathrm{g}$ of protein) for StAR detection, microsomal fractions $(30 \mu \mathrm{g}$ of protein) for aromatase detection and tissular extracts $(50 \mu \mathrm{g}$ of protein) for $\mathrm{ER} \alpha$ detection were run on SDS-PAGE (10-12\%; Bio-Rad Mini Protean 3; Bio-Rad Laboratories, Inc.) $)^{(30)}$ and transferred onto polyvinylidene difluoride membranes (Immobilon-PS2,
Millipore Corporation) in Tris-glycine transfer buffer with $20 \%$ ethanol in a miniblotter (Bio-Rad Laboratories, Inc.). After being blocked in 5\% non-fat dry milk in Tris-buffered saline solution (200 mm-Tris and $1.5 \mathrm{M}-\mathrm{NaCl}, \mathrm{pH} 7 \cdot 4$ ) and $0 \cdot 1 \%$ Tween 20 for $1 \mathrm{~h}$ under shaking, the membranes were incubated with various antibodies at $4^{\circ} \mathrm{C}$ overnight. The antibodies against StAR (1:1000 dilution), aromatase (1:1000 dilution) and $\operatorname{ER} \alpha$ (1:200 dilution) were used for immunoblotting.

A horseradish peroxidase-labelled donkey anti-rabbit IgG against StAR and aromatase or anti-mouse IgG against ER $\alpha$ (1:2000 dilution; Santa Cruz Biotechnology) were used as secondary antibodies (incubation for $1 \mathrm{~h}$ at room temperature). Immunoreactivity was detected using a chemiluminescence kit (ECL Plus, Amersham Biosciences) and a chemiluminescence detector (Fusion FX7, Thermo Fisher Scientific). To normalise the total amount of protein per lane, the membranes were stripped and incubated with a monoclonal goat antibody against $\beta$-actin for StAR (1:1000 dilution; Santa Cruz Biotechnology) or a monoclonal chicken antibody against GAPDH for aromatase and ER $\alpha$ (1:1000 dilution; Millipore). Peroxidase-labelled donkey antigoat IgG (1:2000 dilution; Santa Cruz Biotechnology) or peroxide-labelled donkey anti-chicken IgG (1:2000 dilution; Santa Cruz Biotechnology) were used as secondary antibodies. Densitometric analysis of the intensity of the Western blot bands was carried out using the Image J1.44p software (Freeware; nih.gov).
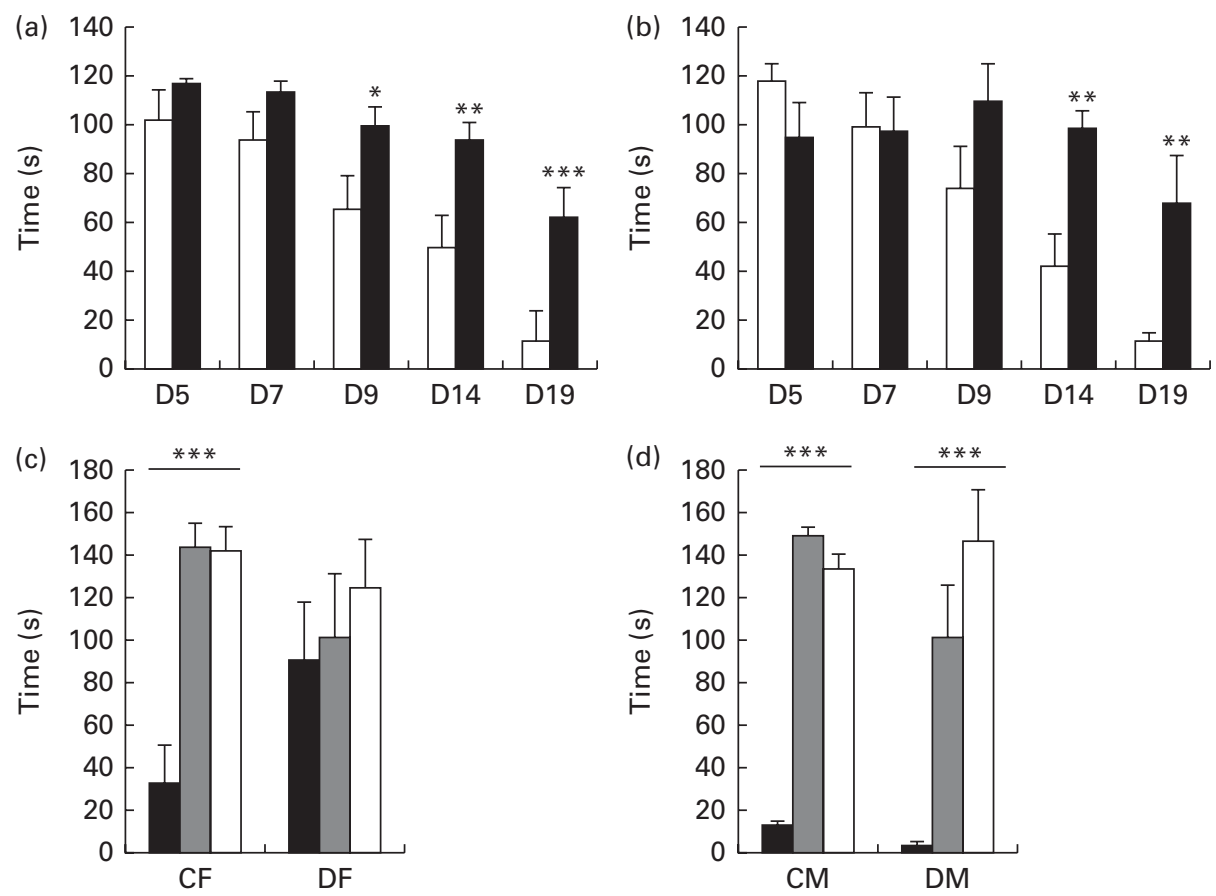

Fig. 1. Behavioural evaluation of olfaction between postnatal days 5 and 19. Time required for (a) female pups and (b) male pups to reach the home cage at postnatal days 5 (D5), 7 (D7), 9 (D9), 14 (D14) and 19 (D19). CM ( $\square$ ), control males ( $n$ 12); DM ( $\square$ ), deficient males ( $n$ 10); CF ( $\square$ ), control females ( $n$ 12); DF ( $\square$ ), deficient females $(n 17)$. Values are means, with standard deviations represented by vertical bars. Mean values were significantly different from the control pups: ${ }^{\star} P<0.05,{ }^{* *} P<0.01,{ }^{\star * *} P<0.001$ (ANOVA). Time spent in each zone of the corridor by (c) female pups and (d) male pups. $\square$, Toluene zone (tol); $\square$, water zone (water); $\square$, central zone (central). ANOVA summary: for CF, $n 12, F(2,24)=21 \cdot 2$, and ${ }^{\star * \star} P<0.0001$ for tol $v$. central and tol $v$. water; for DF, $n 8, F(2,21)=0 \cdot 199$, and $P>0.5438$ for tol $v$. central and tol $v$. water; for $\mathrm{CM}, n 12, F(2,45)=104 \cdot 0$, and ${ }^{\star * \star} P<0.0001$ for tol $v$. central and tol $v$. water; for $\mathrm{DM}, n 12, F(2,24)=22.4$, and ${ }^{* \star *} P<0.0001$ for tol $v$. central and tol $v$. water. 


\section{Quantitative real-time RT-PCR analysis}

After grinding rat brain tissues in liquid $\mathrm{N}_{2}$, total RNA was isolated using the RNeasy Lipid Tissue Mini Kit (Qiagen) following the manufacturer's instructions. RNA content was quantified by spectrophotometry at $260 \mathrm{~nm}$ (Microdrop, multiskan GO, X7, Thermo Fisher Scientific) and analysed by $2 \%$ agarose gel electrophoresis. The first complementary DNA strand was reverse-transcribed from total RNA $(1 \mu \mathrm{g})$ using the QuantiTect Reverse Transcription Kit (Qiagen) following the manufacturer's instructions.

Real-time PCR was carried out on the Lightcycler 2.0 Instrument (Roche Diagnostics) using the QuantiTect SYBR Green PCR Kit (Qiagen). Specific amplification of mRNA coding for StAR, P450 aromatase and ER $\alpha$ was carried out with specific pairs of primers. For StAR mRNA, 5'-AGGAAAGCCAGCAGGAGAAT- $3^{\prime}$ and $5^{\prime}$-CTGTCCATGGGCTGGTCTA- $3^{\prime}$ were used as the forward and reverse primers, respectively. For aromatase mRNA, 5'-TTGATTTTCGCTGAGAGACG-3' and 5'-ACAGAGTGACGGACATGGTG-3' were used as the forward and reverse primers, respectively. For ER $\alpha$ mRNA, 5'-ATGTGTCCAGCTACAAACCAA- $3^{\prime}$ and $5^{\prime}$-CGTATCCCGCCTTTCATC- $3^{\prime}$ were used as the forward and reverse primers, respectively. The internal control, a $90 \mathrm{bp}$ product of polymerase II, was amplified using the forward primer $5^{\prime}$-GCATTAACATCAGGAACAATAAAGGC- $3^{\prime}$ and the reverse primer $5^{\prime}$-GATCTCTCTAAAGTTGACCTCATTGG- $3^{\prime}$. Temperature cycling consisted of one cycle to activate Taq DNA polymerase $\left(95^{\circ} \mathrm{C} ; 15 \mathrm{~min}\right)$, followed by forty-three cycles, each consisting of denaturation $\left(95^{\circ} \mathrm{C}\right.$; $10 \mathrm{~s})$, annealing $\left(53^{\circ} \mathrm{C}\right.$ for StAR and $56^{\circ} \mathrm{C}$ for both $\mathrm{P} 450$ aromatase and $\mathrm{ER} \alpha ; 15 \mathrm{~s})$, and extension $\left(72^{\circ} \mathrm{C} ; 10 \mathrm{~s}\right)$ steps. Melting curve analyses were carried out by raising the temperature from 65 to $95^{\circ} \mathrm{C}$. Cycle threshold $\left(C_{\mathrm{t}}\right)$ was determined for each sample, and real-time PCR amplification efficiencies were obtained by calculating the ratio of crossing points of amplification curves, using the RelQuant software (Roche Diagnostics).

\section{Statistical analysis}

Data were prospectively collected and analysed using the SAS software (SAS Institute, Inc.). Raw data reported as means and standard deviations were compared using one-way ANOVA with Fisher's test. A $P$ value $<0.05$ was considered to indicate statistical significance.
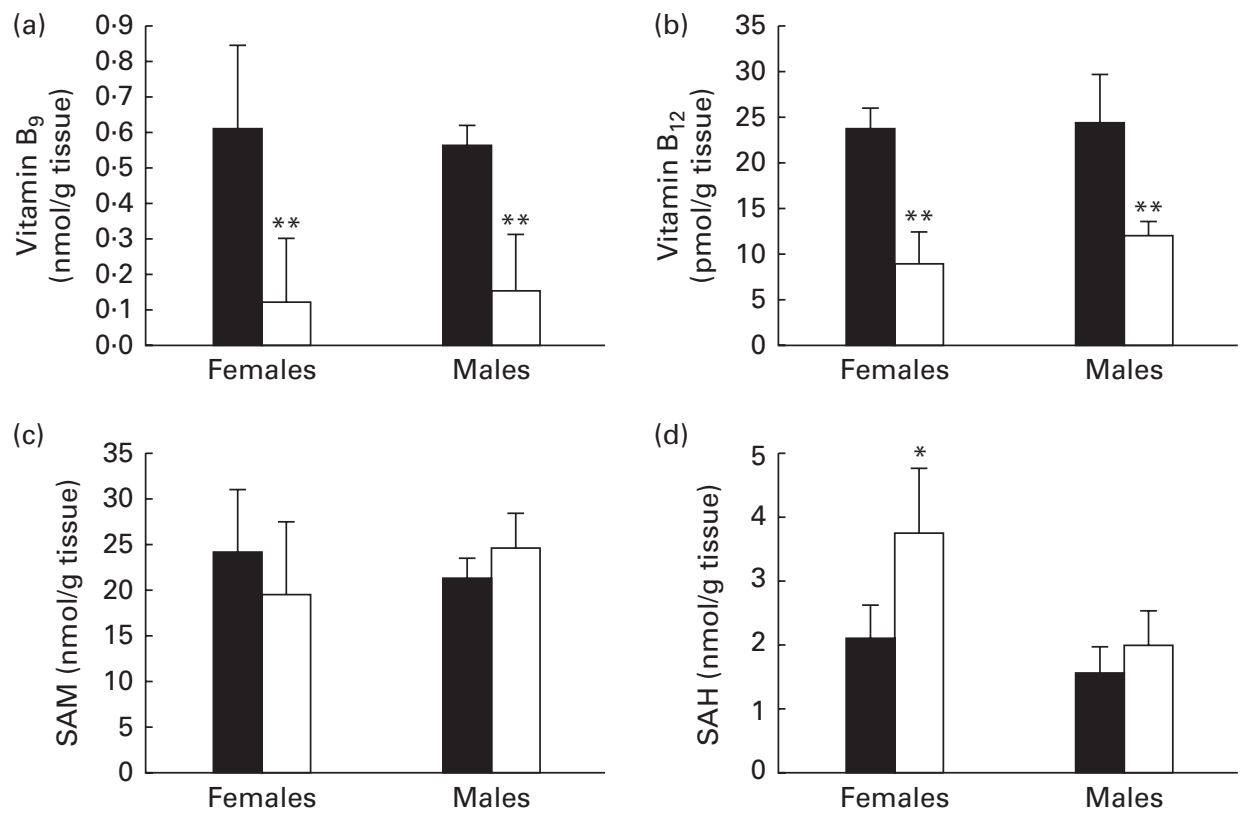

(d)
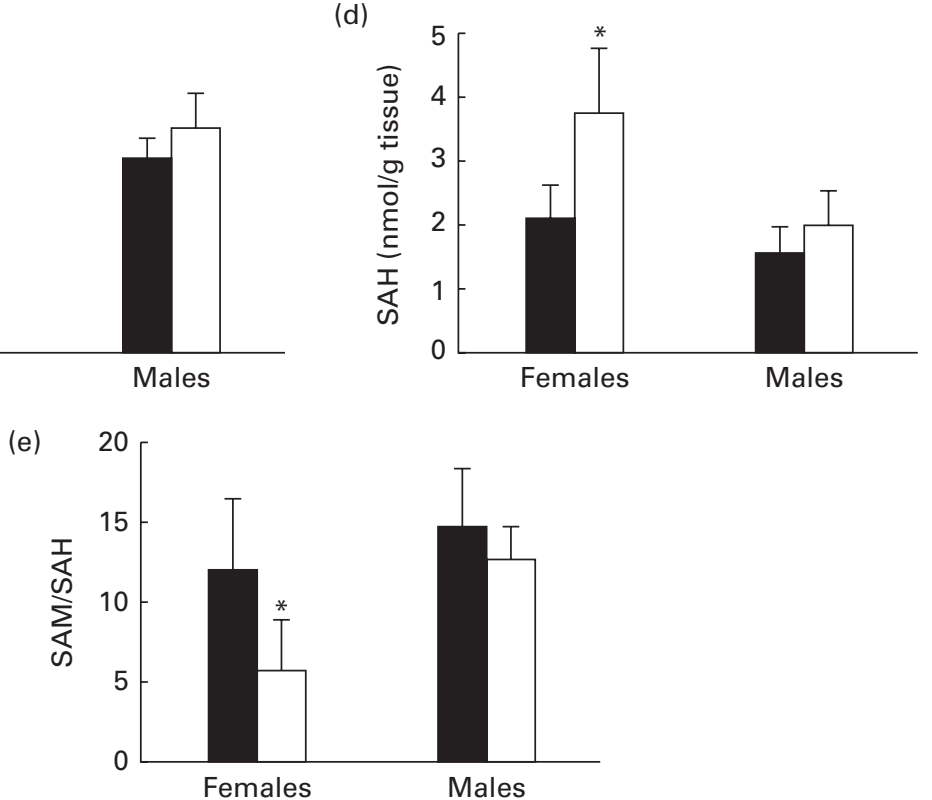

Fig. 2. Concentrations of (a) vitamin $B_{9}$, (b) vitamin $B_{12}$, (c) $S$-adenosylmethionine (SAM), and (d) S-adenosylhomocysteine (SAH) and (e) SAM:SAH ratio in olfactory bulbs of 21 -d-old rat pups. Values are means, with standard deviations represented by vertical bars, $n 5$ per group. Mean values were significantly different from the control pups: ${ }^{\star} P<0.05,{ }^{\star \star} P<0.01$ (ANOVA). $\square$, Control; $\square$, methyl donor-deficient. 


\section{Results}

\section{Effect of the methyl-donor-deficient diet on the body weight of newborn rats}

During weaning, the body weight of female and male pups born to the MDD diet-fed dams was lower than that of pups born to the normal diet-fed dams (Table 1). The difference in weight between pups of the normal diet fed-dams and those of the MDD diet-fed dams was significantly higher $(P<0.01)$ in the females $(56.4 \%)$ than in the males $(44.3 \%)$. A smaller size of the MDD pups was also recorded, as reported previously ${ }^{(5)}$.

\section{Olfactory discrimination behavioural tests}

The newborn rats were subjected to olfactory discrimination tests. Fig. 1(a) and (b) shows that the olfactory nest recognition ability of the deficient pups was affected irrespective of the sex. The acquisition of olfactory cues to return to their own nest was revealed by the shorter time taken to complete the test by both the control male and female pups between postnatal days 5 and 19 (from up to $100 \mathrm{~s}$ to less than $20 \mathrm{~s}$ ). Despite a drop being achieved only by the end of the suckling period (postnatal day 19) in the deficient pups, the time necessary to complete the test remained higher than that in the control pups, starting at postnatal day 9 for the females and postnatal day 14 for the males.
Fig. 1(c) and (d) also shows that the discrimination ability for a repulsive odorant, i.e. toluene, was significantly affected in the deficient female pups than in the control pups. Such a difference was not recorded in the male pups. The control female pups spent only $10 \%$ of their time near the toluene source compared with the $90 \%$ that they spent away from it $(F(2,24)=21 \cdot 2 ; P<0 \cdot 0001)$. By contrast, female pups born to the MDD diet-fed dams spent up to $28 \%$ of their time, evenly distributed among the three zones of the corridor, in the toluene zone $(F(2,21)=0.199 ; P>0.5438)$. By contrast, no sizeable differences were recorded in the male pups, since both the control and deficient pups succeeded in the discrimination test by spending only 5 and $2 \%$ of their time in the toluene zone, respectively $(F(2,45)=104 \cdot 0 ; P<0 \cdot 0001$ and $F(2,24)=22 \cdot 4$; $P<0 \cdot 0001$, respectively).

\section{S-Adenosy/methionine:S-adenosylhomocysteine ratio and vitamin concentrations in olfactory bulbs}

The male and female pups born to the MDD diet-fed dams exhibited significantly lower concentrations of folate (Fig. 2(a)) and vitamin $\mathrm{B}_{12}$ (Fig. 2(b)) in the OB. The measurements of the determinants of Hcy metabolism indicated that, irrespective of the sex, there was no substantial variation in the concentrations of SAM (Fig. 2(c)). Yet, the concentrations of SAH were significantly higher in the $\mathrm{OB}$ of the

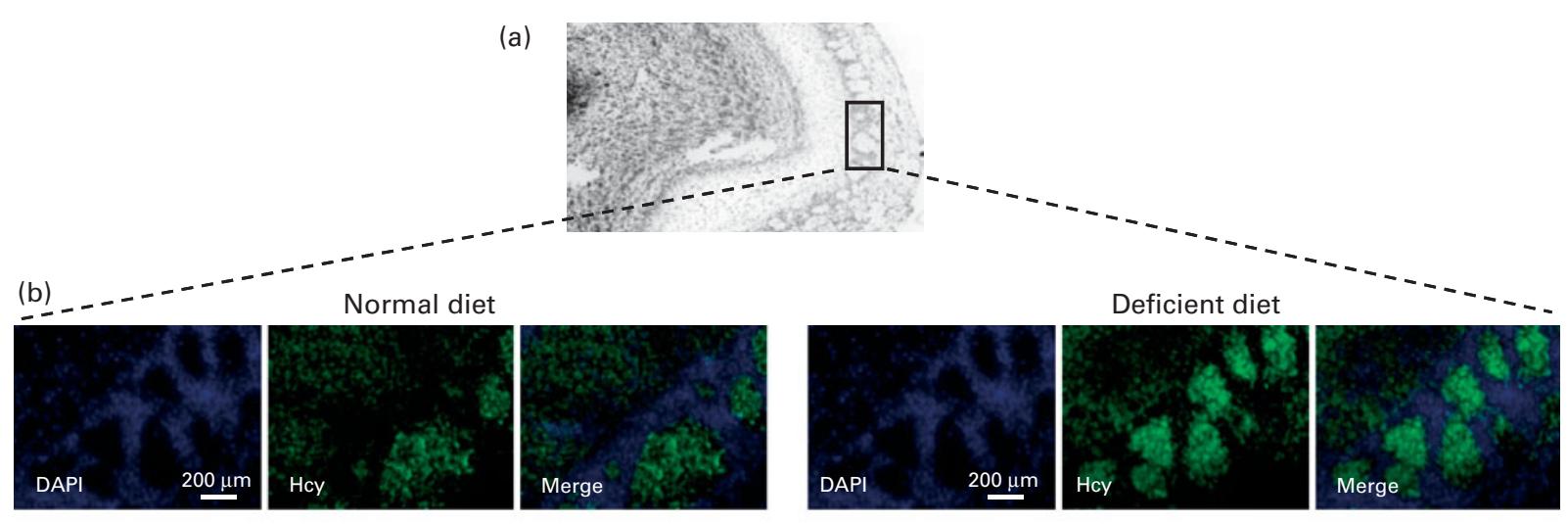

(c)
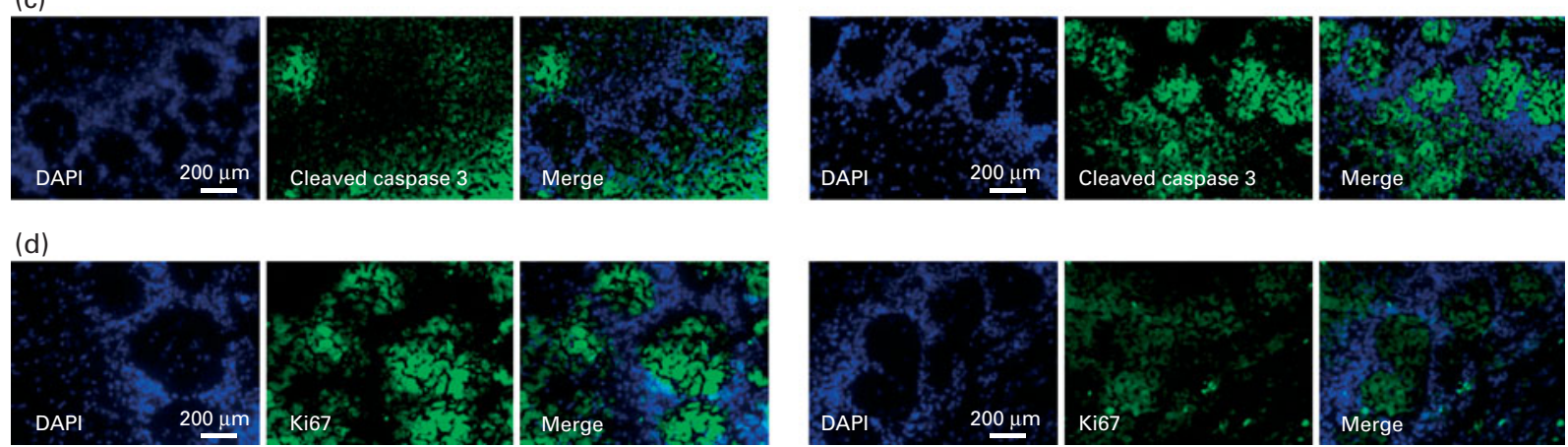

Fig. 3. Immunostaining of homocysteine (Hcy), cleaved caspase 3 and Ki67 in the olfactory bulbs (OB) of 21-d-old female pups. (a) Image of the whole OB representing the glomerular cell layer. (b) Immunostaining of Hcy (green), (c) cleaved caspase 3 (green) and (d) Ki67 in the sagittal sections of OB. The cell nuclei of glomerular cells were stained with the fluorescent dye 4,6-diamidino-2-phenylindole (DAPI, blue). While the immunostaining of cleaved caspase 3 was more elevated, reflecting increased apoptosis in the $\mathrm{OB}$ of the methyl-donor-deficient female pups than in those of the control pups, labelling of Ki67 was almost undetectable, suggesting decreased cell proliferation. $n 5$ per group. Calibration bars $=200 \mu \mathrm{m}$. Original magnification $\times 20$. 
MDD female pups than in those of the control female pups (Fig. 2(d)). The SAM:SAH ratio was consequently lower in the $\mathrm{OB}$ of the MDD female pups (Fig. 2(e)). Furthermore, global DNA methylation was reduced by $19 \cdot 34 \%$ in the $\mathrm{OB}$ of the deficient pups compared with the levels in the control female pups. No variation was observed between the control and MDD male pups.

\section{Homocysteine accumulation, apoptosis and cell} proliferation in the olfactory bulbs of young rats

Hcy was detected in the glomerular cell layer of OB (Fig. 3(a)) and more specifically in the basal and apical dendrites. A strong accumulation of Hcy was observed in the OB of female pups born to the MDD diet-fed dams than in those of pups born to the normal diet-fed dams (Fig. 3(b)). This was not observed in the brain sections of the male pups (data not shown). The labelling of OB cells with a specific indicator of apoptosis, the antibody against cleaved caspase 3, revealed a low immunoreactivity in the glomerular cells of 21-d-old control female pups. It was dramatically higher in the OB sections of the deficient female pups (Fig. 3(c)). Cell proliferation was evaluated using the anti-Ki-67 antibody. As expected, the immunoreactivity of $\mathrm{Ki}-67$ was nearly undetectable in the glomerular cell layer of $\mathrm{OB}$ of female pups born to the MDD diet-fed dams when compared with that in the pups born to the normal diet-fed dams (Fig. 3(d)).
Expression of steroidogenic acute regulatory protein, aromatase, and oestrogen receptor $\alpha$ and immunostaining of oestradiol

Immunohistochemical staining with anti-StAR (Fig. 4(a)), antiaromatase (Fig. 4(b)) and anti-ER $\alpha$ (Fig. 4(c)) antibodies revealed the most intense immunoreactivity in the basal and apical dendrites of the glomerular cell layer of OB in 21-dold pups. The immunoreactivity of the three proteins was weaker in the OB of the deficient female pups. As a consequence, the immunoreactivity of oestradiol was lower in the glomerular cells of 21-d-old female pups of the MDD diet-fed dams (Fig. 4(d)).

Changes in the expression levels of StAR, aromatase and ER $\alpha$ were confirmed by Western blot analyses. A single band was observed for StAR in the mitochondrial fractions isolated from the OB, as illustrated in Fig. 5(a). Densitometric analysis indicated a substantial decrease in the levels of both mitochondrial StAR protein (Fig. 5(a)) and ER $\alpha$ (Fig. 5(c)) in female pups of the MDD diet-fed dams, which reached $60 \%$ in the case of StAR, when compared the levels in pups of the normal diet-fed dams. A slighter but non-significant decline was observed in the control and deficient male pups. The expression of aromatase protein measured in the microsomal fractions of $\mathrm{OB}$ of the deficient female pups was also significantly lower. No difference was found between the MDD and control male pups (Fig. 5(b)) (a)

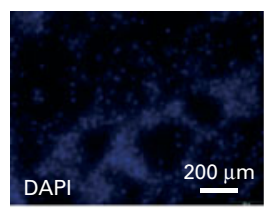

(b)

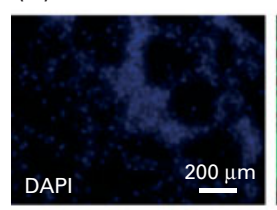

(c)

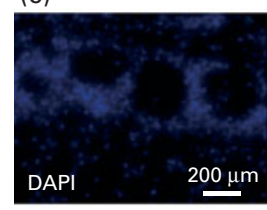

(d)

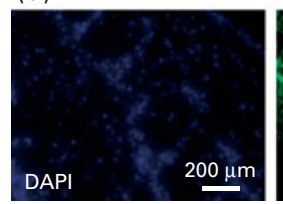

Normal diet
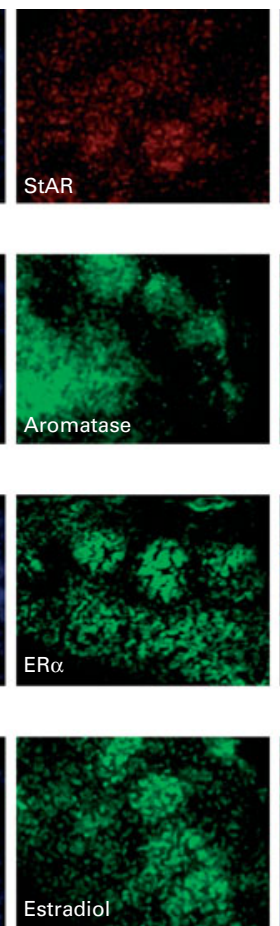
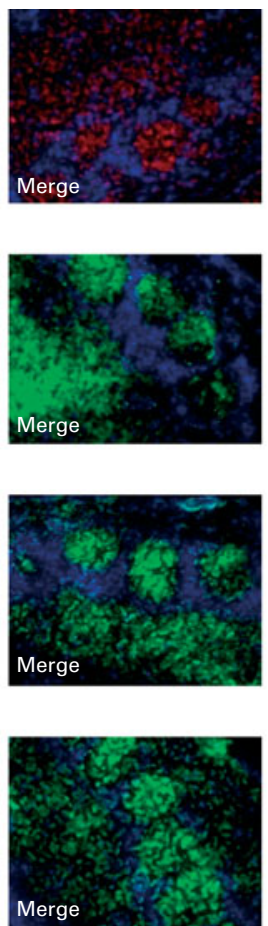
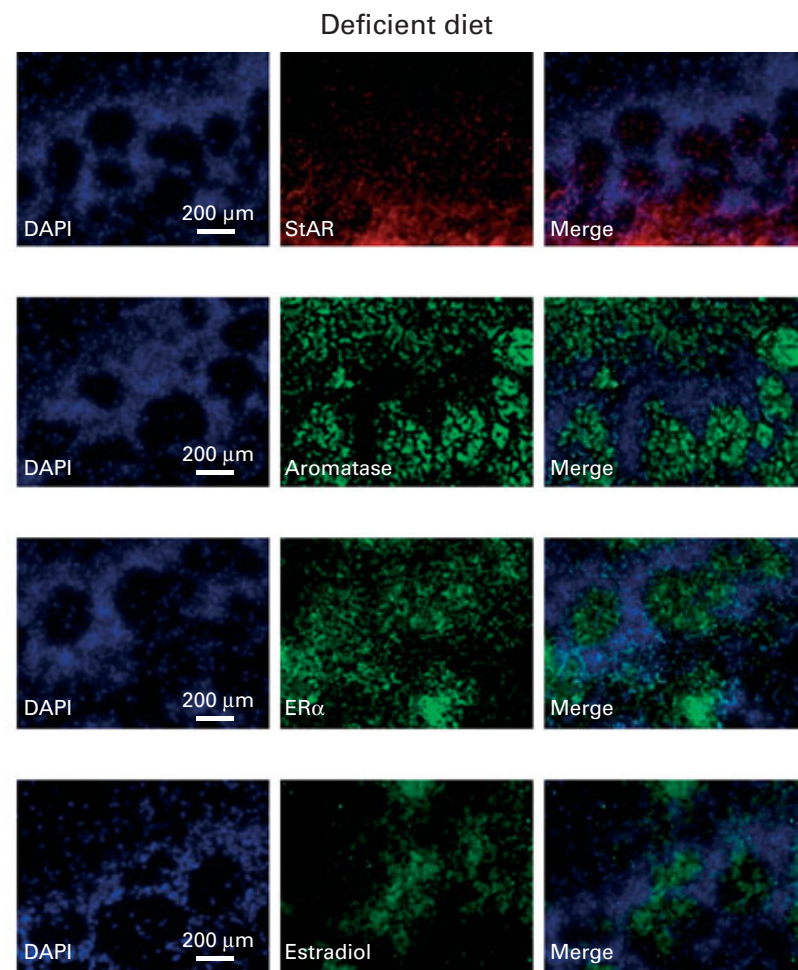

Fig. 4. Immunostaining of steroidogenic acute regulatory protein (StAR), aromatase, oestrogen receptor $\alpha$ (ER $\alpha$ and oestradiol in the olfactory bulbs (OB) of 21-d-old female pups. (a) Immunostaining of StAR (red), (b) aromatase (green), (c) ER $\alpha$ (green) and (d) oestradiol (green) in the sagittal sections of OB. The cell nuclei of glomerular cells were stained with the fluorescent dye 4,6-diamidino-2-phenylindole (DAPI, blue). Immunoreactivity of the three proteins and oestradiol was lower in the $\mathrm{OB}$ of the methyl-donor-deficient female pups than in those of the control pups. $n 5$ per group. Calibration bars $=200 \mu \mathrm{m}$. Original magnification $\times 20$. 

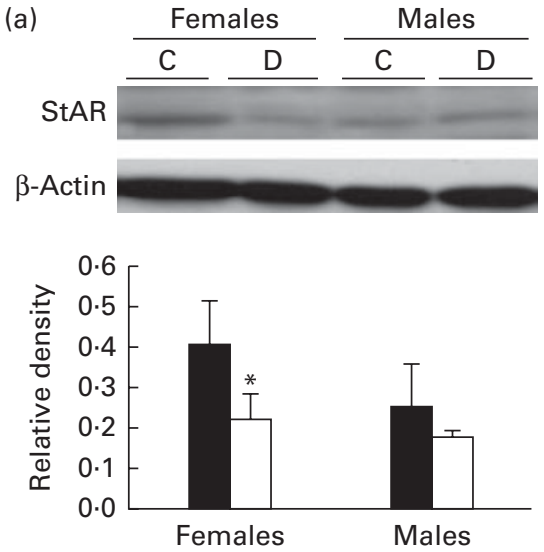

(b)
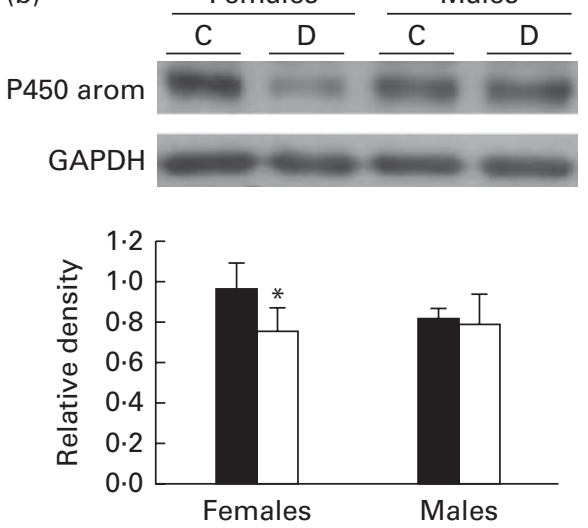
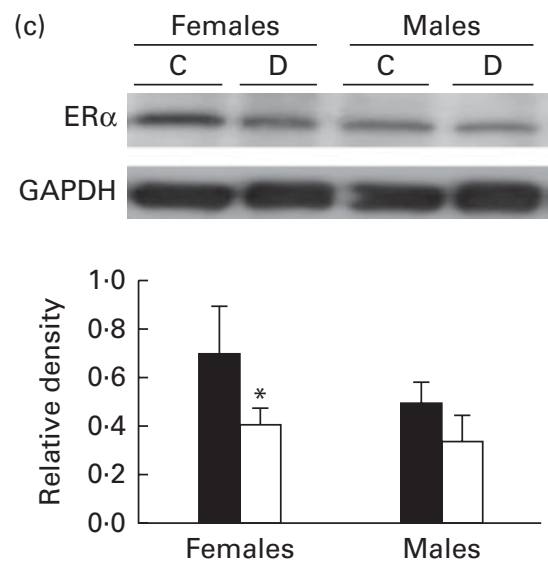

Fig. 5. Effect of the methyl donor-deficient diet on the expression of steroidogenic acute regulatory protein (StAR), aromatase and oestrogen receptor $\alpha$ (ER $\alpha$ proteins in the olfactory bulbs (OB) of 21-d-old pups. (a) Representative set of Western blot showing immunodetectable StAR protein. From left to right, the lanes contain mitochondria from the OB $(30 \mu \mathrm{g}$ protein) of the 21 -d-old control $(C, \mathbf{D})$ and deficient $(\mathrm{D}, \square)$ female and male pups. Expression of StAR (30 kDa) was quantified and normalised against that of $\beta$-actin $(43 \mathrm{kDa})$. (b) Representative set of Western blot showing immunodetectable P450 aromatase protein. The lanes contain microsomes isolated from the $\mathrm{OB}(30 \mu \mathrm{g}$ protein) of the 21 - $\mathrm{d}$-old $\mathrm{C}$ and $\mathrm{D}$ pups. Expression of $\mathrm{P} 450$ aromatase $(55 \mathrm{kDa})$ was quantified and normalised against that of glyceraldehyde 3-phosphate dehydrogenase (GAPDH) (38 kDa). (c) Representative set of Western blot showing immunodetectable ER $\alpha$ protein. The lanes contain proteins from the $\mathrm{OB}(50 \mu \mathrm{g}$ protein) of the $21-\mathrm{d}$-old $\mathrm{C}$ and $\mathrm{D}$ pups. Expression of $\mathrm{ER} \alpha(66 \mathrm{kDa})$ was quantified and normalised against that of GAPDH. Densitometric data were obtained from five separate experiments ( $n 5$ per group). Values are means, with standard deviations represented by vertical bars. * Mean values were significantly different from the control pups $(P<0.05 ;$ ANOVA).

To examine the consequences of MDD on gene expression, the mRNA levels of StAR, aromatase and $E R \alpha$ were quantified in the OB of 21-d-old rat pups by quantitative RT-PCR analysis. There was no significant difference in the levels of the three proteins in the deficient male pups when compared with the levels in the control pups (Fig. 6). The mRNA level of StAR varied in the same manner as protein contents, and the mRNA expression of StAR measured in 21-d-old female pups born to the MDD diet-fed dams was significantly lower than that in female pups born to the normal diet-fed dams (Fig. 6(a)). Similarly, the mRNA expression of aromatase (Fig. 6(b)) and ER $\alpha$ (Fig. 6(c)) in the OB of the deficient female pups was lower.

\section{Discussion}

Pups born to the MDD diet-fed dams exhibited lower body weight and impaired olfactory discrimination when compared with those born to the normal diet-fed dams. The exposure to the MDD diet during gestation and lactation was associated with significantly lower concentrations of vitamins $B_{9}$ and $\mathrm{B}_{12}$ in the $\mathrm{OB}$ of 21 -d-old pups. Vitamin $\mathrm{B}_{12}$ acts as a cofactor of the methionine synthase enzyme, which is involved in the synthesis of methionine, the precursor of SAM, which is the universal methyl donor. SAM is transmethylated into SAH and Hcy originates from SAH hydrolysis. Thus, it is easily understandable that a lack of nutritional intake of $\mathrm{B}$ vitamins will affect Hcy metabolism ${ }^{(5)}$. The concentrations of the classical determinants of Hcy metabolism have been reported previously for whole-brain homogenates, and enhanced concentration of total SAH associated with unchanged SAM concentrations have been measured ${ }^{(6)}$. In the present study, we demonstrated for the first time that the concentrations of $\mathrm{SAH}$ were significantly higher in the $\mathrm{OB}$ of only female rats, with a consecutively reduced index of methylation capacity represented by the SAM:SAH ratio. This is in accordance with the lower amounts of methylated DNA quantified in the OB of the MDD female pups.

Hcy is a potent neurotoxic and pro-apoptotic compound, and its accumulation in neuronal cells has been reported 
to be associated with neurodegenerative diseases and with cognitive defects in the elderly ${ }^{(2)}$. An association of cerebral accumulation of Hcy with functional defects as well as the hallmarks of apoptosis in hippocampal neurogenic areas has been reported previously ${ }^{(6,7)}$. Sustained Hcy immunoreactivity, concomitantly with both an increasing number of cells undergoing apoptosis and an impairment of cell proliferation in the glomerular cell layer of $\mathrm{OB}$ of the deficient pups, suggests that MDD also affects the $\mathrm{OB}$ of newborn rats.

We have recently hypothesised that MDD occurring in early life could be associated with altered synaptic plasticity and
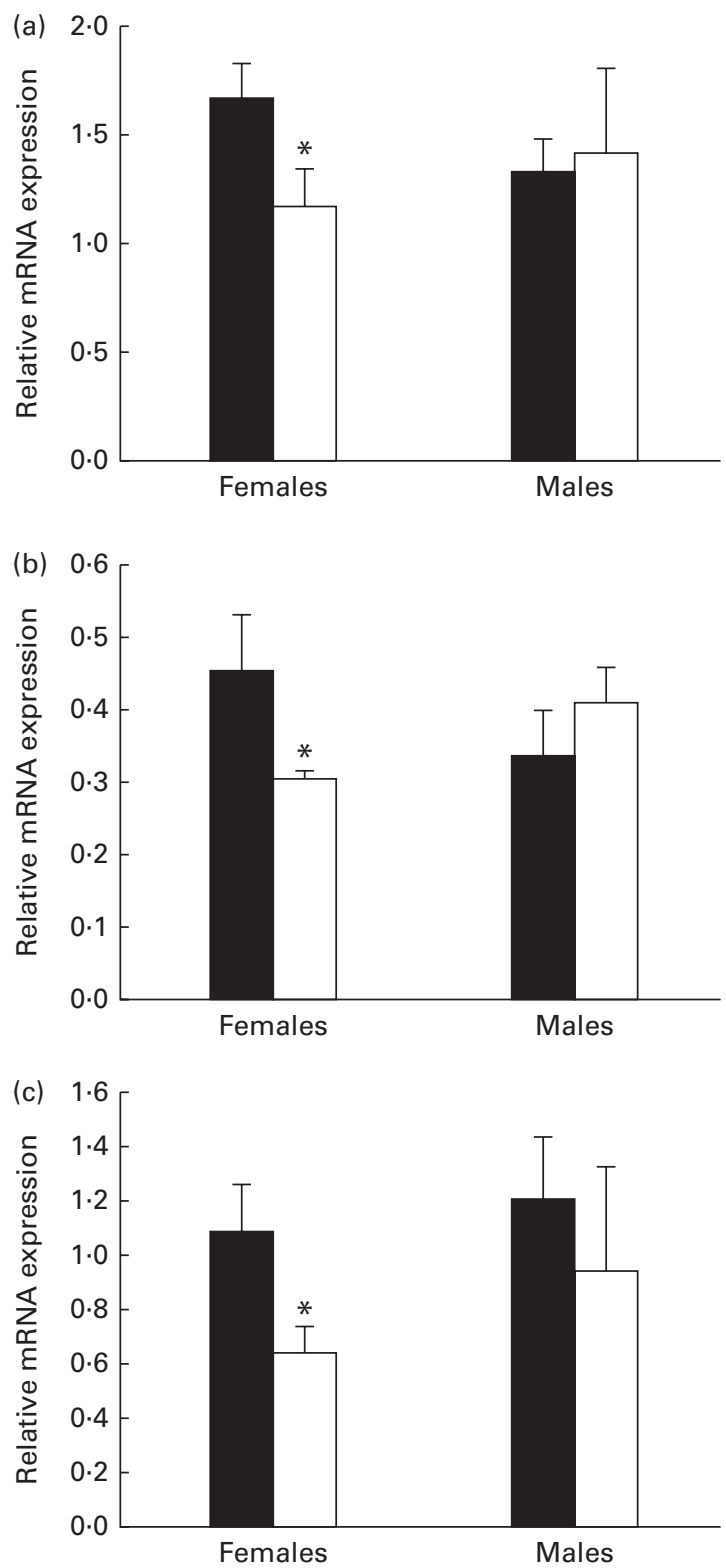

Fig. 6. Results of the quantitative RT-PCR analysis of the effect of the methyl donor-deficient diet on the expression of (a) steroidogenic acute regulatory protein (StAR), (b) aromatase and (c) oestrogen receptor $\alpha$ (ER $\alpha$ mRNA in the olfactory bulbs of 21-d-old female rats. Arbitrary unit refers to an internal standard in the olfactory bulbs of the control $(\square)$ and deficient $(\square)$ pups ( $n 5$ per group, run in duplicate). Values are means, with standard deviations represented by vertical bars. * Mean values were significantly different from the control pups $(P<0.05 ;$ ANOVA). neurogenesis ${ }^{(3)}$. The postnatal period is crucial as neurogenesis is achieved around the third postnatal week in rats ${ }^{(31)}$, which corresponds to the age of the pups assessed in the present study. During this period, high concentrations of ER are present in $\mathrm{OB}$, whereas $E R \alpha$ transcripts are undetectable in adulthood $^{(32)}$. Moreover, the early postnatal period is characterised by a strong effect of oestrogens on neurogenesis and survival of $\mathrm{OB}$ neurons, whereas in adulthood oestrogens control both the survival of neurons and their functional integration $^{(33)}$. In the present study, we demonstrated that MDD impaired the critical steps of the neurosteroidogenic pathway, specifically only in female rats, by decreasing both the protein and transcription levels of StAR, aromatase and $\mathrm{ER} \alpha$ in the OB. The reduced detection of oestradiol in the glomerular cell layer of $\mathrm{OB}$ appears to be a consequence of these changes and strengthens the hypothesis that the decrease in the expression levels of StAR, aromatase and $\mathrm{ER} \alpha$ leads to poor neurosteroidogenesis in the $\mathrm{OB}$ of the deficient pups. These harmful effects of MDD on the synthesis of cerebral steroids are not surprising, since previous studies have reported an association between folate and steroid metabolism. In this respect, preconception folate deficiency affects fertility and follicular response to ovarian stimulation in association with depleted levels of oestradiol. According to the authors, this could result from the impaired methylation of aromatase $\mathrm{DNA}^{(34)}$.

The behavioural evaluation of olfactory performance strongly suggests that the impaired neurosteroidogenesis in OB consecutively to gestational MDD could have functional consequences. If olfactory nest recognition ability is affected irrespective of the sex during the third week after birth, the 21-d-old deficient female pups would specifically exhibit a dramatically reduced odour discrimination performance. The T-maze test evaluates home-odour recognition and is based on both the olfactory and locomotor abilities of pups. Considering that both the male and female deficient pups displayed body-weight loss, it can be hypothesised that the mean time to complete the test would be lower in the MDD pups than in the control pups. Thus, we carried out the placepreference test, which is specifically based on olfactory functions. Indeed, a lack of clear discrimination is specific in pathophysiological damage affecting the neuro-olfactory tissues $^{(35,36)}$. The learning of odour identification is essential not only during the postnatal period but also in adulthood, since clinical studies have reported less olfactory sensitivity ${ }^{(37)}$ and fewer $\mathrm{OB}$ volumes in depressive patients ${ }^{(38)}$.

In addition to the greater weight difference between the MDD and control pups recorded in females than in the males, the patent functional and tissular consequences of MDD appeared to be sex dependent. The effects of oestradiol depend on various factors, especially strain and $\operatorname{sex}^{(39,40)}$. A sexual dimorphic effect of oestrogens on the development of dendritic spines in cultured $\mathrm{OB}$ with significant effects of oestradiol treatment on primary cultures derived from brains of female rats has been reported ${ }^{(41)}$. Interestingly, a previous study has revealed that neonatal repeated handling results in a loss of maternal odour preference, specifically in female rat pups than in control pups ${ }^{(42)}$. These dimorphic variations 
seem to be associated with steroid-dependent molecular mechanisms that occur via the nuclear receptors and co-activators of steroids, and most of them are present at high levels in the OB of rats according to a sex-specific localisation, so as to regulate brain development and behaviour ${ }^{(43)}$.

In conclusion, the present results provide the first evidence of the sex-dependent deleterious effects of perinatal MDD on olfactory discrimination performance associated with impaired neurosteroidogenesis, since the decreased expression levels of key proteins result in an apparent reduced content of oestradiol in the $\mathrm{OB}$ of 21 -d-old female rat pups. This may contribute to their growth retardation and body-weight loss through impaired suckling. It has been shown that an early folate privation has not only developmental effects, but also life-long effects ${ }^{(44)}$. Whether it is associated with neuropsychological disorders in adulthood requires further investigation.

\section{Acknowledgements}

The authors thank Professor D. Stocco (Texas Tech University, USA) for providing antibodies against StAR protein.

The present study received institutional grants from the French National Agency for Research (ANR Nutrivigene project) and the Region of Lorraine (France). ANR Nutrivigene and the Region of Lorraine had no role in the design and analysis of the study or in the writing of this article.

The authors' contributions are as follows: S. E. H. C, G. P., J.-L. D., J.-L. G. and B. L.-M. conceived and designed the experiments; S. E. H. C., G. P., N. M. and J.-M. A. carried out the experiments; S. E. H. C., G. P., J.-L. D., J.-L. G. and B. L.-M. analysed the data; S. E. H. C., G. P., J.-L. D., J.-L. G. and B. L.-M. wrote the article.

None of the authors has any conflicts of interest.

\section{References}

1. Obeid R \& Herrmann W (2005) Homocysteine, folic acid and vitamin B-12 in relation to pre- and postnatal health aspects. Clin Chem Lab Med 43, 1052-1057.

2. Tchantchou F (2006) Homocysteine metabolism and various consequences of folate deficiency. J Alzheimers Dis 9 , 421-427.

3. Daval JL, Blaise S \& Guéant JL (2009) Vitamin B deficiency causes neural cell loss and cognitive impairment in the developing rat. Proc Natl Acad Sci U S A 106, E1.

4. Craciunescu CN, Brown EC, Mar MH, et al. (2004) Folic acid deficiency during late gestation decreases progenitor cell proliferation and increases apoptosis in fetal mouse brain. J Nutr 134, 162-166.

5. Blaise S, Alberto JM, Nédélec E, et al. (2005) Mild neonatal hypoxia exacerbates the effects of vitamin-deficient diet on homocysteine metabolism in rats. Pediatr Res 57, 777-782.

6. Blaise SA, Nédélec E, Schroeder H, et al. (2007) Gestational vitamin B deficiency leads to homocysteine-associated brain apoptosis and alters neurobehavioral development in rats. Am J Pathol 170, 667-679.

7. Blaise SA, Alberto JM, Audonnet-Blaise S, et al. (2007) Influence of preconditioning-like hypoxia on the liver of developing methyl-deficient rats. Am J Physiol Endocrinol Metab 293, E1492-E1502.
8. Blaise SA, Nédélec E, Alberto JM, et al. (2009) Short hypoxia could attenuate the adverse effects of hyperhomocysteinemia on the developing rat brain by inducing neurogenesis. Exp Neurol 216, 231-238.

9. Garcia MM, Guéant-Rodriguez RM, Pooya S, et al. (2011) Methyl donor deficiency induces cardiomyopathy through altered methylation/acetylation of PGC-1 $\alpha$ by PRMT1 and SIRT1. J Pathol 225, 324-335.

10. Pooya S, Blaise S, Moreno Garcia M, et al. (2012) Methyl donor deficiency impairs fatty acid oxidation through PGC- $1 \alpha$ hypomethylation and decreased ER- $\alpha$, ERR- $\alpha$, and HNF- $4 \alpha$ in the rat liver. $J$ Hepatol 57, 344-351.

11. Bossenmeyer-Pourié C, Blaise S, Pourié G, et al. (2010) Methyl donor deficiency affects fetal programming of gastric ghrelin cell organization and function in the rat. Am J Pathol 176, 270-227.

12. Kojima M \& Kangawa K (2005) Ghrelin: structure and function. Physiol Rev 85, 495-522.

13. Leon M (1992) Neuroethology of olfactory preference development. J Neurobiol 23, 1557-1573.

14. Lledo PM \& Saghatelyan A (2005) Integrating new neurons into the adult olfactory bulb: joining the network, lifedeath decisions, and the effects of sensory experience. Trends Neurosci 28, 248-254.

15. Sullivan RM \& Wilson DA (2003) Molecular biology of early olfactory memory. Learn Mem 10, 1-4.

16. Shingo T, Gregg C, Enwere E, et al. (2003) Pregnancy-stimulated neurogenesis in the adult female forebrain mediated by prolactin. Science 299, 117-120.

17. Mellon SH (2007) Neurosteroid regulation of central nervous system development. Pharmacol Ther 116, 107-124.

18. Stocco D (2000) The role of the StAR protein in steroidogenesis; challenges for the future. J Endocrinol 164, 247-253.

19. Midzak A, Rone M, Aghazadeh Y, et al. (2011) Mitochondrial protein import and the genesis of steroidogenic mitochondria. Mol Cell Endocrinol 336, 70-79.

20. Furukawa A, Miyatake A, Ohnishi T, et al. (1998) Steroidogenic acute regulatory protein (StAR) transcripts constitutively expressed in the adult rat central nervous system: colocalization of StAR, cytochrome $P$-450SCC (CYP XIA1), and 3beta-hydroxysteroid dehydrogenase in the rat brain. $J$ Neurochem 71, 2231-2238.

21. Lavaque E, Sierra A, Azcoitia I, et al. (2006) Steroidogenic acute regulatory protein in the brain. Neuroscience $\mathbf{1 3 8}$, $741-747$.

22. Garcia-Segura LM (2008) Aromatase in the brain: not just for reproduction anymore. $J$ Neuroendocrinol 20, 705-712.

23. Brock O, Keller M, Veyrac A, et al. (2010) Short term treatment with oestradiol decreases the rate of newly generated cells in the subventricular zone and main olfactory bulb of adult female mice. Neuroscience 166, 368-376.

24. Heth G, Nevo E \& Todrank J (1996) Seasonal changes in urinary odors and in responses to them by blind subterranean mole rats. Physiol Behav 60, 963-968.

25. Jacquot L, Pourié G, Buron G, et al. (2006) Effects of toluene inhalation exposure on olfactory functioning: behavioral and histological assessment. Toxicol Lett 165, 57-65.

26. Buron G, Hacquemand R, Pourié G, et al. (2007) Comparative behavioral effects between synthetic 2,4,5-trimethylthiazoline (TMT) and the odor of natural fox (Vulpes vulpes) feces in mice. Behav Neurosci 121, 1063-1072.

27. Chéry C, Barbé F, Lequere C, et al. (2002) Hyperhomocysteinemia is related to a decreased blood level of vitamin $B_{12}$ in the second and third trimester of normal pregnancy. Clin Chem Lab Med 40, 1105-1108. 
28. Luippold G, Delabar U, Kloor D, et al. (1999) Simultaneous determination of adenosine, $S$-adenosylhomocysteine and $S$-adenosylmethionine in biological samples using solid-phase extraction and high-performance liquid chromatography. J chromatogr B Biomed Sci Appl 724, 231-238.

29. Kerek R, Geoffroy A, Bison A, et al. (2013) Early methyl donor deficiency may induce persistent brain defects by reducing Stat3 signaling targeted by miR-124. Cell Death Dis 4, e755.

30. Laemmli K (1970) Cleavage of structural proteins during the assembly of the head of bacteriophage T4. Nature 227, 680-685.

31. Kim HJ, Park CH, Roh GS, et al. (2002) Changes of steroidogenic acute regulatory protein mRNA expression in post-natal rat development. Dev Brain Res 139, 247-254.

32. Guo XZ, Su JD, Sun QW, et al. (2001) Expression of estrogen receptor (ER) $-\alpha$ and $-\beta$ transcripts in the neonatal and rat cerebral cortex, cerebellum and olfactory bulb. Cell Res 11, 321-324.

33. Veyrac A \& Bakker J (2011) Postnatal and adult exposure to oestradiol differentially influences adult neurogenesis in the main and accessory olfactory bulb of female mice. FASEB J 25, 1048-1057.

34. Twigt JM, Hammiche F, Sinclair KD, et al. (2011) Preconception folic acid use modulates estradiol and follicular responses to ovarian stimulation. J Clin Endocrinol Metab 96, E322-E329.

35. Buron G, Hacquemand R, Pourié G, et al. (2009) Carbon dioxide effects on olfactory functioning: behavioral, histological and immunohistochemical measurements. Toxicol Lett 188, 251-257.

36. Zou J, Pan YW, Wang Z, et al. (2012) Targeted deletion of ERK5 MAP kinase in the developing nervous system impairs development of GABAergic interneurons in the main olfactory bulb and behavioral discrimination between structurally similar odorants. J Neurosci 32, 4118-4132.

37. Atanasova B, Graux J, El Hage W, et al. (2008) Olfaction: a potential cognitive marker of psychiatric disorders. Neurosci Biobehav Rev 32, 1315-1325.

38. Negoias S, Croy I, Gerber J, et al. (2010) Reduced olfactory bulb volume and olfactory sensitivity in patients with acute major depression. Neuroscience 169, 415-421.

39. Bakker J, Honda S, Harada N, et al. (2002) The aromatase knock-out mouse provides new evidence that estradiol is required during development in the female for the expression of sociosexual behaviors in adulthood. $J$ Neurosci 22, 9104-9112.

40. Barker JM \& Galea LA (2008) Repeated estradiol administration alters different aspects of neurogenesis and cell death in the hippocampus of female, but not male, rats. Neuroscience 152, 888-902.

41. Wu Y, Moriya-Ito K, Iwakura T, et al. (2011) Sexually dimorphic effects of estrogen on spines in cultures of accessory olfactory bulb. Neurosci Lett 500, 77-81.

42. Raineki C, De Souza M, Szawka R, et al. (2009) Neonatal handling and the maternal odor preference in rat pups: involvement of monoamines and cyclic AMP response element-binding protein pathway in the olfactory bulb. Neuroscience 159, 31-38.

43. Bian C, Zhang D, Guo Q, et al. (2011) Localization and sex-difference of steroid receptor coactivator-1 immunoreactivities in the brain of adult female and male mice. Steroids 76, 269-279.

44. Hussain N (2012) Epigenetic influences that modulate infant growth, development, and disease. Antioxid Redox Signal 17, 224-236. 